\title{
Simple method for extracting gold from electrical and electronic wastes using hydrometallurgical process
}

\author{
Mohammad Aali Dehchenari ${ }^{1}$, Saeed Hosseinpoor ${ }^{2}$, Rahim Aali $^{3^{*}}$, Nilowfar Salighehdar Iran ${ }^{4}$, Mohsen Mehdipour $^{5}$ \\ ${ }^{1}$ BSc of Mechanic Engineering, Young Researchers and Elite Club, Majlesi Branch, Islamic Azad University, Isfahan, Iran \\ ${ }^{2}$ Instructor of Civil Engineering - Structure, Department of Environmental Health, School of Health, Urmia University of Medical \\ Sciences, Urmia, Iran \\ ${ }^{3}$ Assistant Professor of Environmental Health Engineering, Department of Environmental Health Engineering, Faculty of Nursing and \\ Health, Urmia University of Medical Sciences, Urmia, Iran \\ ${ }^{4} \mathrm{Ph} . \mathrm{D}$ Student of Health Education and Health Promotion, Department of Health Education and Health Promotion, Tehran University \\ of Medical Sciences, Tehran, Iran \\ ${ }^{5}$ Assistant Professor of Environmental Health Engineering, Department of Environmental Health Engineering and Member of \\ Environmental Health Engineering Research Center, Kerman University of Medical Sciences, School of Health, Kerman, Iran
}

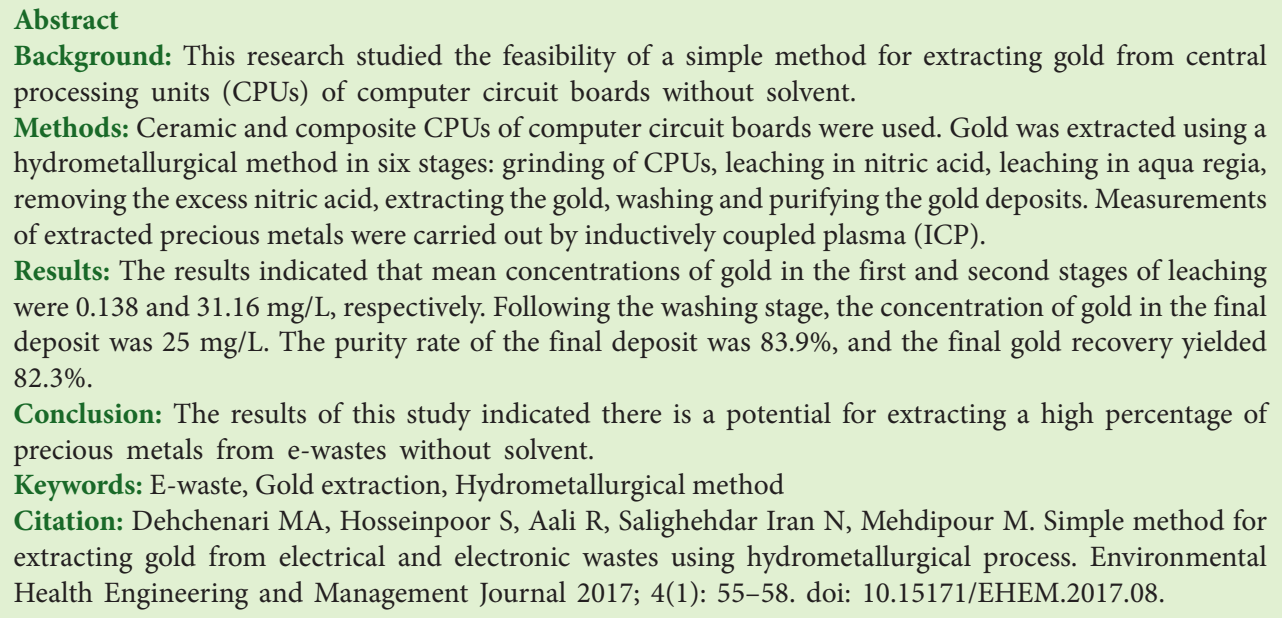

Article History:

Received: 30 September 2016

Accepted: 6 December 2016

ePublished: 29 December 2016

\section{Introduction}

E-waste has been a major segment of the waste produced in the past decades $(1,2)$. The problem of e-waste began with the development of electronic-based industries (3, 4). E-waste comprises $2-5 \%$ of municipal solid wastes (MSW). These wastes include a wide range of discarded electronic products including computers, mobile phones, video players, copying devices and printers, tape recorders, microwaves, refrigerators, and freezers. The An estimated 50 million tons of e-waste was produced globally in 2014 (4). This is 1\%-3\% (1,636 million tons) of the total produced waste around the world (Organization for Economic Corporation and Development [OECD]). Second-hand computers, mobile phones, and televisions made up an estimated 9.8 million tons of e-waste in 2015. In some advanced countries, e-wastes account for over $80 \%$ of municipal waste. Scientific reports have shown that developing countries will produce twice the amount of e-waste of developed countries by 2030 (4).

Recovering precious metals from e-waste through hydrometallurgical processes is more attractive economically than other methods (3). In recent decades, a large amount of research has been conducted on recovering metals from e-wastes, especially precious metals, using hydrometallurgical methods. In comparison with other pyrometallurgical methods, hydrometallurgical methods are more accurate and predictable $(3,5)$. A hydrometallurgical process consists of first a set of operations including acidic or alkaline dissolution (leaching) of solids. The resulting solutions are then exposed to separation and purification methods including deposition, solvent extraction, adsorption, and ion exchange in order to isolate and concentrate the intended metals. Eventually, to recover the metals, the solutions are treated using the processes of electrolyte recovery, chemical recovery, and crystallization (3). Some 
major problems of using a hydrometallurgical process are the unavailability and the prohibitive cost of effective extraction solvents.

The current research studied the feasibility of a simple hydrometallurgical method for extracting gold from ceramic and composite central processing units (CPUs) of computer circuit boards without special solvents.

\section{Materials and methods}

This research utilized composite (15 samples) and ceramic (15 samples) CPUs of computer circuit boards (Figure 1). The study process consisted of six steps (Figure 2). Step 1 (grinding of the CPUs): First, any dust or other particles were removed from the CPUs. This stage aimed to achieve the full contact of acid with the deepest parts and complete dissolution of its metals. The size of the grind pieces was $1 \mathrm{~mm}$. Step 2 (leaching in nitric acid): Concentrated nitric acid (65\%, Merck Co.) was added. Leaching time in this stage was 1 hour. The amount of acid utilized was based on the type of CPU-containing board. To prevent the loss
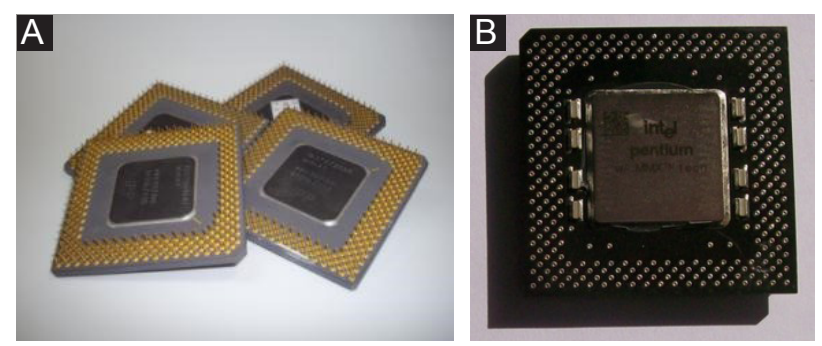

Figure 1. Ceramic (A) and Composite (B) CPUs of computer circuit boards used for gold extraction (Intel and AMD brands)

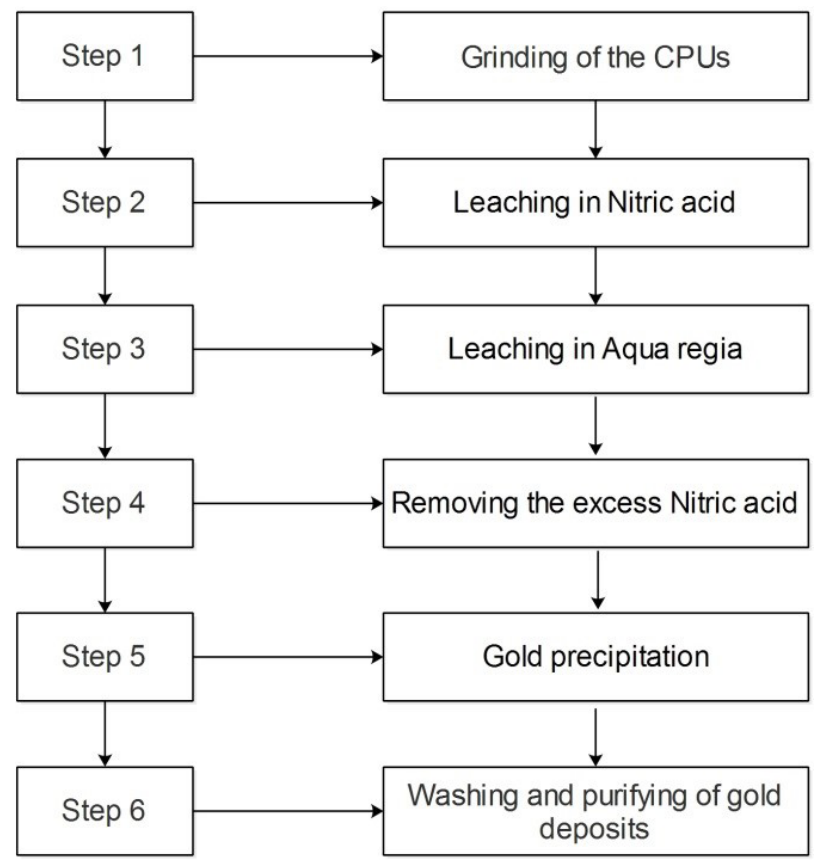

Figure 2. Six steps used to extract gold from computer circuit boards. of gold, boiling was avoided (6). Step 3 (leaching in aqua regia): The aqua regia solution was obtained using three volumes of $\mathrm{HCl}$ plus one volume of $\mathrm{HNO}_{3}$ (under the hood). Typically, the gold of composite CPU-containing boards is easily dissolved in 4-to-1 aqua regia. The reaction releases bubbles and is followed by sublimation of $\mathrm{NO}_{2}$ brown gas. The solution was decanted, and the pieces were subsequently washed with $10 \mathrm{~mL}$ of concentrated $\mathrm{HCl}$ so that all of the gold content was fed into the solution. Step 4 (removing the excess nitric acid): Sulfuric acid was added to the solution to accelerate the removal of nitric acid and sedimentation of the lead as lead sulfate (if present). The time required for the complete removal of nitric acid from this solution was 45 minutes. At this stage, the solution's color was golden yellow and the solution's $\mathrm{pH}$ was around 0.5 . If any deposit was observed (possibly related to silver chloride), it was separated by a filter. Step 5 (precipitation of gold): A certain amount of iron sulfate (per one gram of gold: $4.2 \mathrm{~g}$ of iron sulfate) was dissolved in warm water and gradually added to the gold-containing solution. For better dissolution of iron sulfate, some drops of $\mathrm{HCl}$ can be used. Step 6 (washing and purifying gold deposits): The deposit was coated with $\mathrm{HCl}$ and then boiled (to dissolve the excess copper sulfate). Following boiling, the acid was removed and the deposit was re-washed in $\mathrm{HCl}$ to ensure the cleanness of the gold powder. Eventually, the deposit was filtered and washed with large amounts of warm water. At this stage, the deposit was placed in an oven at a temperature of $100^{\circ} \mathrm{C}$ to dry (7). Sampling and measuring of precious metals were performed following the first and second stages of leaching, after the stage of gold extraction, and finally following the purification of deposits. The value of precious metals was measured by inductively coupled plasma (ICP-OES, Perkin Elmer, Optima 7300DV).

\section{Results}

Analysis results from the first stage leaching (nitric acid leaching) indicated that the average amount of gold metal was $0.138 \mathrm{mg} / \mathrm{L}$. No platinum was found, but silver had the greatest amount (Figure $3 \mathrm{~A}$ ). ICP analysis values of the second leaching (aqua regia leaching) are presented in Figure 3B. As expected, the deposit washing stage should have had the lowest value of the investigated metals. The values of $\mathrm{Ag}, \mathrm{Au}, \mathrm{Cu}$, and $\mathrm{Pt}$ were 1.174, 0.04, 3.668 $(\mathrm{mg} / \mathrm{L})$, and ND, respectively. These results show that a large amount of the metals were recovered in the previous steps. The results obtained from ICP analysis of the achieved deposit showed the weight of the final deposit following drying was $20.42 \mathrm{mg}$. The purity of the obtained deposit was $83.9 \%$, and the recovery yield was $82.3 \%$. The values are shown in Figure 3C.

\section{Discussion}

Different researchers have indicated that effective recovery of precious metals (especially gold) from e-wastes is 

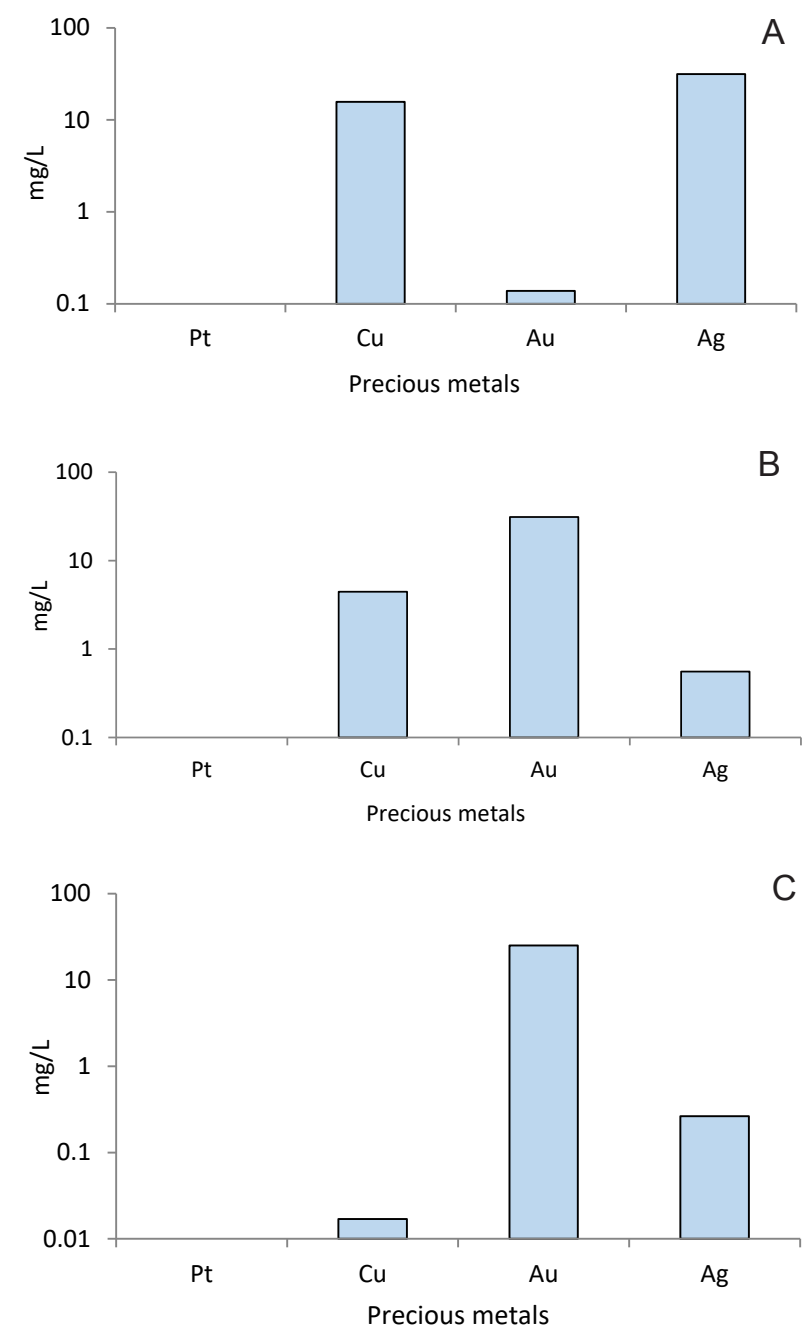

Figure 3. Average elements from first leaching step (A), Average elements from second leaching step (B) and Average elements from precipitation step (C).

feasible $(7,8)$. Optimum recovery in the present study was $82.3 \%$. Other researchers achieved higher recovery rates using special solvent. Quinet et al managed to achieve 95\% recovery of gold from a leaching solution (step one, oxidative sulfuric acid; step two, thiourea leach; step three, recovery of gold with activated carbon) in their proposed method (9). Kogan et al succeeded in extracting 98\% gold from a leaching solution using a solvent extraction method (10). In the process presented by Biong et al, over 99.9\% of gold was extracted from a leaching solution using solvent (Dibutyl carbinol) extraction method. Park and Fray indicated that using solvent extraction method (toluene as extraction solution and tetra-octyl ammonium bromide as phase transfer agent), gold with a purity of $97 \%$ can be extracted (6). The use of oxidant agents (electrogenerated chlorine) and resin recovery (Amberlite XAD7HP) of gold from mobile phones was found to achieve a maximum recovery of $95 \%$ (11).

Researchers who have achieved high percentages of gold recovery from leaching solution have based extraction on solvent extraction methods. However, due to a lack of access to this type of special solvents, no solvent extraction stage was considered in the current study. Nevertheless, the results suggest that all percentages of gold recovery can be achieved by optimizing processes and fully implementing the proposed flowchart (Figure 2).

\section{Conclusion}

Electrical and e-wastes possess substantially important sources of different metals, especially precious metals. Extraction of these metals can both decrease the pressure on natural resources and reduce environmental contamination. The quantitative and qualitative investigation of precious metals extraction on a wider scale by considering technological aspects can bring about a direction for developing precious metals extraction technology.

\section{Acknowledgments}

The authors would like to thank the staff of Isfahan Science and Technology Town.

\section{Ethical issues}

The authors have completely observed ethical issues including plagiarism, misconduct, data fabrication, and redundancy.

\section{Competing interests}

The authors declare that no competing interests exist.

\section{Authors' contributions}

All authors contributed equally to the study, and all authors participated in data acquisition, analysis, and interpretation. All authors critically reviewed, refined, and approved the manuscript.

\section{References}

1. Widmer R, Oswald-Krapf H, Sinha-Khetriwal D, Schnellmann M, Böni H. Global perspectives on e-waste. Environ Impact Assess Rev 2005; 25(5): 436-58. doi: 10.1016/j.eiar.2005.04.001.

2. Behnamfard A, Salarirad MM, Veglio F. Process development for recovery of copper and precious metals from waste printed circuit boards with emphasize on palladium and gold leaching and precipitation. Waste Manag 2013; 33(11): 2354-63. doi: 10.1016/j.wasman.2013.07.017.

3. Cui J, Zhang L. Metallurgical recovery of metals from electronic waste: a review. J Hazard Mater 2008; 158(2-3): 228-56. doi: 10.1016/j.jhazmat.2008.02.001.

4. Baldé K. The Global E-waste Monitor 2014: Quantities, Flows and Resources. Bonn, Germany: United Nations University; 2015. Available from: https://i.unu.edu/media/ unu.edu/news/52624/UNU-1stGlobal-E-Waste-Monitor2014-small.pdf.

5. Kang HY, Schoenung JM. Electronic waste recycling: a review of U.S. infrastructure and technology options. Resources, Conservation and Recycling 2005; 45(4): 368400. doi: 10.1016/j.resconrec.2005.06.001. 
6. Park YJ, Fray DJ. Recovery of high purity precious metals from printed circuit boards. J Hazard Mater 2009; 164(2-3): 1152-8. doi: 10.1016/j.jhazmat.2008.09.043.

7. Syed S. Recovery of gold from secondary sources-A review. Hydrometallurgy 2012; 115-116: 30-51. doi: 10.1016/j.hydromet.2011.12.012.

8. Sum EY. The recovery of metals from electronic scrap. JOM 1991; 43(4): 53-61. doi: 10.1007/BF03220549.

9. Quinet P, Proost J, Van Lierde A. Recovery of precious metals from electronic scrap by hydrometallurgical processing routes. Minerals and Metallurgical Processing 2005; 22(1): 17-22.

10. Kogan V. Recovery of precious metals from electronic scrap by hydrometallurgical processing. Google Patents; 2006. http://www.google.com/patents/WO2006013568A2?cl=en.

11. Kim EY, Kim MS, Lee JC, Pandey BD. Selective recovery of gold from waste mobile phone PCBs by hydrometallurgical process. J Hazard Mater 2011; 198: 206-15. doi: 10.1016/j. jhazmat.2011.10.034. 